\title{
Dermatological Conditions Associated with Pregnancy: A Hospital Based Study
}

\author{
Pandey $\mathrm{S}^{1}$, Sharma $\mathrm{N}^{2}$, Pokhrel $\mathrm{K}^{3}$
}

\begin{abstract}
Background: Pregnancy Changes can be both physiologic and pathologic, affecting commonly the skin, nails, and hair shafts. Pregnancy has immunologic, endocrine, metabolic and vascular changes, which leads to changes of skin and its appendages and can affect every organ of pregnant women including the skin. Pregnancy also modifies the course of a number of preexisting dermatological conditions. Objectives: The aim of our study was to study the common skin manifestation during pregnancy. Material and Method: Hundred pregnant women were enrolled in the study. This is a hospital- based prospective cross- sectional descriptive study, conducted in the out-patient department (OPD) of Department of Dermatology and Venereology and Department and Obstetrics and Gynecology at Nepalgunj Medical College Teaching Hospital Kohalpur, Banke Nepal, conducted over a period of two year, June 2016 to May 2018. A detailed history along with complete cutaneous examination was carried out in all patients. Relevant investigations were done wherever necessary. Result: A total of 100 pregnant women were recruited in our study from June 2016 to May 2018 .Out of these, 65(65\%) were primigravidas and 35(35\%) were multigravidas. Their age range was 18 to 37 years with the mean of 24 . Most of them presented in the third trimester. Pruritus was the commonest symptom accounting for $28(28 \%)$ cases. Physiological changes were seen in $75(75 \%)$ cases and $10(10 \%)$ cases of specific dermatoses of pregnancy were seen. Conclusion: Skin manifestations are quite common in pregnancy and physiological changes were frequently seen. This study emphasizes the need for a scrupulous and meticulous search for dermatological and sexually transmitted diseases instead of a casual cursory examination and clinicians need to distinguish between physiological skin changes and specific dermatoses of pregnancy for better patient care.
\end{abstract}

Key words: Cutaneous changes, Pregnancy

\section{INTRODUCTION}

Pregnancy has immunologic, endocrine, metabolic and vascular changes which leads to changes of skin and its appendages and can affect every organ of pregnant women including the skin. Some of these changes are due to de novo production of a variety of protein and steroid hormones by the fetoplacental unit as well as the increased activity of maternal pituitary, thyroid and adrenal glands. Recognition of these changes is important for proper classification and appropriate treatment ${ }^{1}$. Pregnancy changes can be both physiologic and pathologic, affecting commonly the skin, nails, and hair shafts. Moreover, pregnancy modifies the course of a number of preexisting dermatological conditions ${ }^{2}$. Previously normal skin

1. Dr. Sumit Pandey

2. Dr. Nirmala Sharma

3. Dr. Kumar Pokhrel

Address for correspondence:

Dr. Sumit Pandey

Assistant Professor

Department of Dermatology

Nepalgunj Medical College \& Teaching Hospital

Kohalpur, Banke, Nepal

Email:sumitpandey207@yahoo.com may show changes, preexisting dermatoses may become aggravated, improved or be unaffected ${ }^{3}$. Lab investigations are required when the diagnosis remains in question despite a careful history and thorough physical examination ${ }^{1}$. Dermatoses of pregnancy is divided into three categories which includes physiological skin changes, skin diseases affected by pregnancy and specific dermatoses of pregnancy ${ }^{4}$.There is a group of inflammatory dermatoses specifically related to pregnancy. A condition like pemphigoid gestation is, polymorphic eruption of pregnancy is specific to pregnancy.

Our study aimed to examine the common skin manifestation during pregnancy in women coming for antenatal checkup at Nepalgunj Medical College Teaching Hospital Kohalpur and therefore have a working knowledge of both physiological and pathological cutaneous lesions during pregnancy.

\section{MATERIALS AND METHOD}

This is a hospital based prospective cross- sectional study. The study was conducted in the outpatient department (OPD) of Department of Dermatology and Obstetric and Gynecology at Nepalgunj Medical College Teaching Hospital Kohalpur Nepal. The study was conducted over a period of two year, June 2016 to May 2018. The study population comprised patients attending the department of Dermatology and Venereology 
and Department of Obstetric and Gynecology of the NGMCTH Kohalpur Banke. One hundred pregnant women attending the Outpatient department were included in the study. All pregnant women irrespective of age, parity presenting with skin manifestation were included. Altogether 100 patients were included irrespective of their dermatological complains and all of their skin manifestations were recorded. The diagnosis based on detailed history, clinical features, clinical examinations, and appropriate investigations. Diagnosis was essentially clinical in most cases. Diagnostic laboratory investigations were performed when required and where clinical diagnosis was difficult. Detailed history included chief complaints (if present) related to skin, presence of itching, skin lesions, onset in relation to duration of pregnancy, jaundice, vaginal discharge, past or family history of similar lesions, exacerbating factors, associated medical or skin disorders etc. was elicited and recorded. Relevant systemic examination was carried out. If any preexisting skin disease was present, any evidence of exacerbation or remission was recorded. All statistical analysis was performed using the SPSS 20 and Microsoft excel 2010 software program. Data were tabulated in terms of age, parity, trimester, different skin disorders.

\section{RESULTS}

A total of 100 pregnant women were included in our study from June 2016 to May 2018 .Out of these, 65 (65\%) were primigravidas and $35(35 \%)$ were multigravidas (FIGURE 1). Their age range was 18 to 37 years (TABLE 1 ) with the mean of 24. Most of them presented mainly in the third trimester.

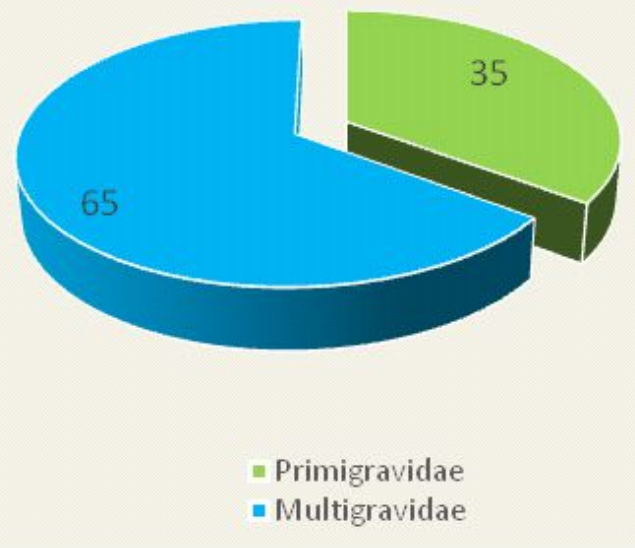

Figure 1: Parity Index of Pregnant Women

\begin{tabular}{|l|l|l|}
\hline $\begin{array}{l}\text { Age Distribution } \\
\text { (Years) }\end{array}$ & Numbers & Percentages \\
\hline $15-20$ & 8 & 8 \\
\hline $21-25$ & 53 & 53 \\
\hline $26-30$ & 33 & 33 \\
\hline $31-35$ & 5 & 5 \\
\hline $36-40$ & 1 & 1 \\
\hline Total & 100 & 100 \\
\hline
\end{tabular}

Table I: Age distribution of pregnant women

\section{Clinical manifestation in pregnant woman}

Pruritus was the commonest symptom accounting for $28(28 \%)$ cases. Physiological changes were seen in 75(75\%) cases and 11 (11\%) cases of specific dermatoses of pregnancy were seen. Other dermatoses affected by pregnancy were seen in 35 cases (35\%)(Figure 2). Out of 3 patients who suffered from sexually transmitted diseases, 2 had condyloma acuminata and 1 had latent syphilis.

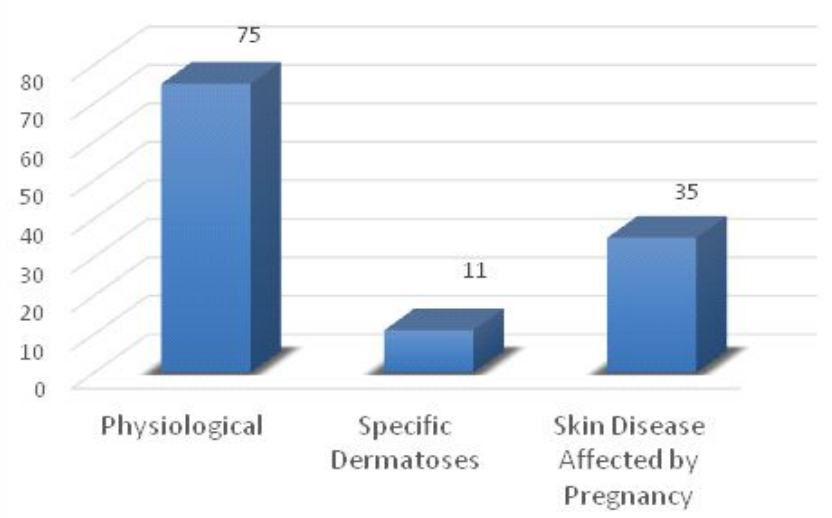

Figure 2: Type of skin condition in pregnant women

\section{Physiological skin changes}

Among the physiological skin changes observed, most common were pigmentary changes (41 cases) including melasma, linea nigra, areolar hyperpigmentation and hyperpigmentation of the skin (Figure 3). Melasma were seen in 32 cases, among which malar (80\%) were the frequent presentation (Figure 4 and 5). In this study the vascular changes were seen in 15 cases (15\%). Out of which non-pitting edema was the commonest 11 cases (11\%) (Table II) 


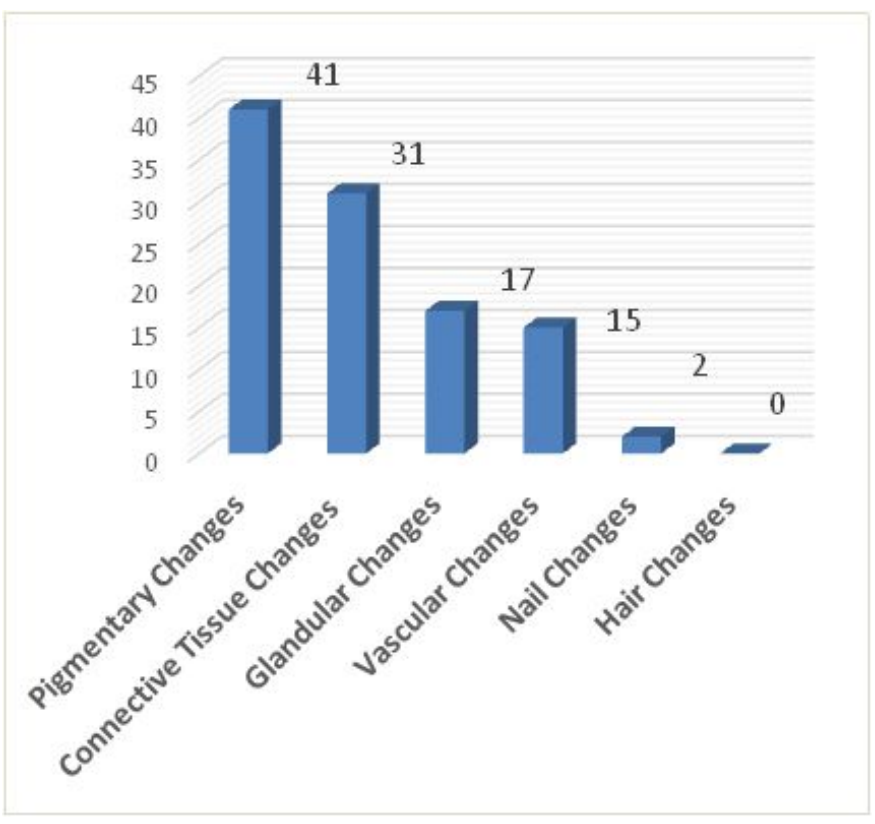

Figure 3: Pattern of physiological changes

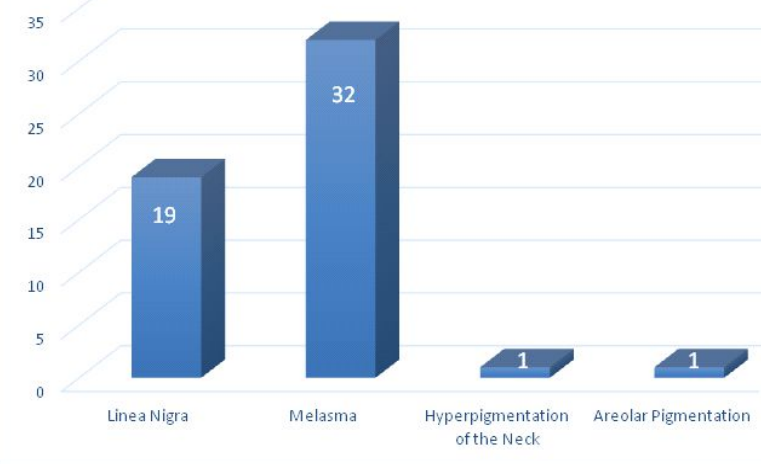

Figure 4: Pattern of pigmentary changes

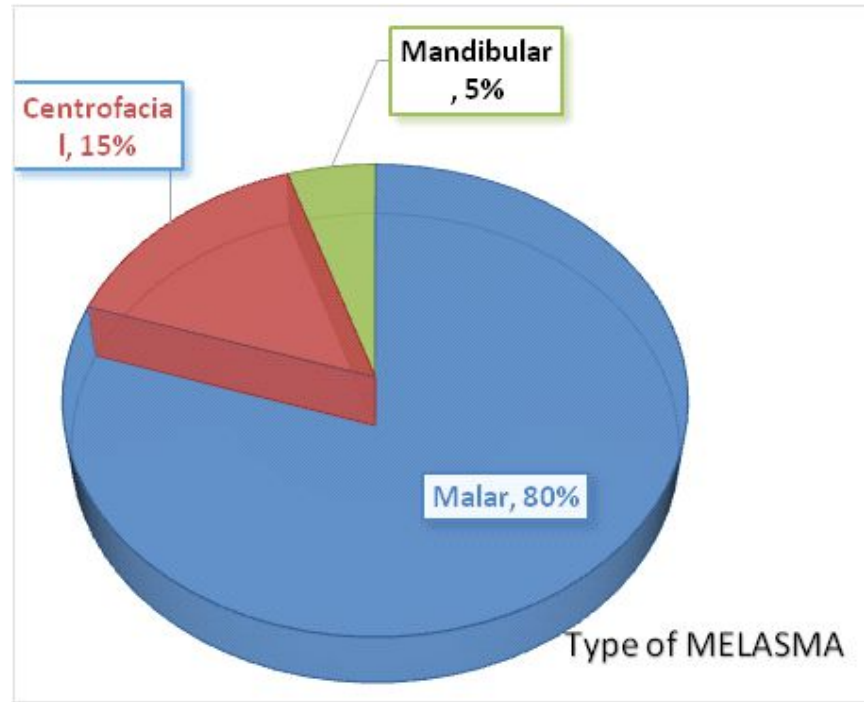

Figure 5: Pattern of Melasma

\begin{tabular}{|l|l|}
\hline Type of Vascular Changes & Frequency \\
\hline Non Pitting Edema of the Legs & 10 \\
\hline Varicosity & 2 \\
\hline Spider Telagiectasia & 1 \\
\hline Jacquemier-Chadwick Sign & 1 \\
\hline Goodell's Sign & 1 \\
\hline TOTAL & 15 \\
\hline
\end{tabular}

Table II: Pattern of Vascular Changes

\section{Specific dermatoses of pregnancy}

Out of 100 pregnant women seen during the study, 10 pregnant women had specific dermatoses of pregnancy. In this study, 5 were found to have pruritic urticarial papules and plaques of pregnancy. Out of these 4 were primigravidae and 1 were multigravidae. Three cases of pruritis gravidarum were seen in this study. Out of these 2 were primigravidas and 1 was multigravidas. In all these 3 cases liver function tests were normal expect for raised alkaline phosphatase. One case of pruritic folliculitis of pregnancy and she was a primigravida. Two cases of prurigo of pregnancy and 2 cases of atopic eczema of pregnancy were seen (Figure 6).

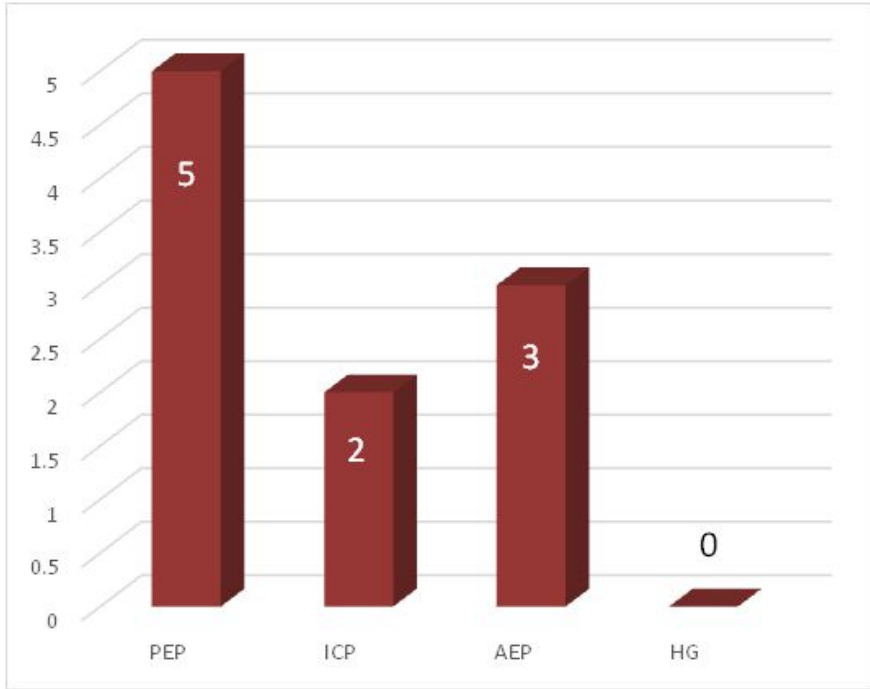

Figure 6: Pattern of Specific Dermatoses

\section{Dermatoses affected by pregnancy}

Pruritus was the commonest symptom accounting for 28 cases (28\%). Among this infection were the commonly seen dermatoses. Out of 14 cases (14\%) of infection, fungal infection was the commonest ( 6 cases) (Table III). 


\begin{tabular}{|l|l|}
\hline Type of INFECTION & Frequency \\
\hline Bacterial & 1 \\
\hline Fungal & 6 \\
\hline Viral & 4 \\
\hline Infestation & 3 \\
\hline TOTAL & 14 \\
\hline
\end{tabular}

Table III: Pattern of Infection

\section{DISCUSSION}

Pruritus was the commonest symptom accounting for $28 \%$ and $7 \%$ cases had itching without any skin changes. According to Winton et al, pruritus from all causes may occur in $17 \%$ of pregnant women ${ }^{2}$. In our study the pruritus was due to dermatophytic infection, scabies, eczema and urticaria and contact dermatitis besides the pregnancy specific dermatoses which underscores the need for a meticulous search for the underlying disorder. Patients complaining of white discharge per vagina, candidiasis accounted for $1 \%$ of the cases. In contrary to our study, candidal vaginitis occurs 10 to 20 times more frequently during pregnancy according to Dotz et al and the view is shared by Winton et $a l^{2,5}$. Out of 3 patients who suffered from sexually transmitted diseases, 2 had condylomata acuminata and 1 had latent syphilis. In the study by Raj et al the incidence of syphilis was $0.9 \%$. This emphasizes the need for routine serological screening for syphilis in all pregnant women ${ }^{6}$.

In this study pigmentary changes occurred in 41 (41\%) cases which included melasma (32\%) 32cases, linea nigra seen was 19 cases (19\%), hyperpigmentation of the neck was seen in $1 \%$ cases and areolar pigmentation was seen in $1 \%$ cases. Pigmentary changes occurred in $98.82 \%$ of the patients in a study done by Shivakumar et $a l^{7}$. Melasma was observed in $10.58 \%$ similar to the finding of Raj et al $^{6}$ Literature incidence of melasma in white skin is reported between 50 to $70 \%{ }^{2,8}$.

Wong Rc et $\mathrm{al}^{8}$ reported $90 \%$ cases of striae distensae and in this study only $31 \%$ had striae distensae in which 20 cases were primigravidae and 11 cases were multigravidae. The onset was commonly seen during the third trimester .The most common site seen in primigravidas was lower abdomen and pink shiny striae whereas multigravidas showed mostly white atrophic striae. Striae is uncommon in Asian and African-American women and there seems to be a familial tendency ${ }^{1}$. Poindevin et $a l^{9}$ in 1959, observed that women with lighter complexions had a greater tendency to develop striae compared with women of darker complexions.

In this study no pregnant women gave any history of hair changes and only 2 complained of brittle nail. In a study done by Rashmi Kumari et $a l^{4}$, among 607 women, 11 gave a history of increased hair loss and only 5 patients noticed lengthening and improvement in their scalp hair, whereas 591 (97.4\%) gave history of no change in hair density. Muzaffar et $a{ }^{10}$ reported hair changes in $18(12.8 \%)$ cases. Out of those 18 cases, diffuse thinning of scalp hair was noted in 7 (38.9\%) cases. Nine $(50 \%)$ patients noticed lengthening and improvement in their scalp hair. Frontoparietal recession and hypertrichosis was seen in one case each. Increased appearance of Montgomery's tubercles is well known during pregnancy in $30-50 \%$ of pregnant women ${ }^{11}$ In this study, montgomery's tubercles were seen in 15 cases (15\%).

Vascular changes result from distention, instability and proliferation of vessels and regress postpartum. Non pitting edema of legs, eyelids, face and hands is present in about $50 \%$ of women during the third trimester ${ }^{2}$. The edema decreases during the day and is thought to be due to secondary sodium and water retention in conjunction with increased capillary permeability ${ }^{11}$.Vascular changes seen in Rashmi Kumari et al study included nonpitting edema of feet in 59 (9.8\%) cases and abdominal wall edema in 3 cases ${ }^{4}$. In this study the vascular changes were seen in 15 cases (15\%). Out of which non pitting edema was the commonest 10 cases(10\%),varicosity was seen in 2 cases, in which 1 pregnant women had varicose of the lower legs and one with varicocele of the vulva. Varicosities are most common in anus and legs, appearing in $40 \%$ of pregnant women during the 3 rd trimester ${ }^{2}$ Raj et al ${ }^{6}$ noted varicose veins in 6 out of 1,175 women. Vascular spiders were seen in 2 cases. Vascular changes in mucosa was not frequently in this study. Only 1 case of Jacquemier-Chadwick sign and Goodell's sign were seen. Esteve et al ${ }^{12}$ observed vascular changes in 50 women including vascular spiders in 32 out of the 60 women. Out of 14 cases of infection seen, dermatophytic infection (6 cases) were common, 3 cases of scabies, and 4 cases of viral infection. 2 case of varicella was seen in third trimester, 1 cases of condylomata accuminata, 1 cases of molluscum contagiosum. Scabies was recorded in $17.64 \%$ of the cases and was the commonest condition recorded ${ }^{7}$.

Specific dermatoses of pregnancy are almost always associated with pruritus and an eruption of variable severity. Holmes and Black $^{13}$ proposed a simplified clinical classification of the specific dermatoses of pregnancy. This classification basically 
subdivided the specific dermatoses of pregnancy into four groups: (i) pemphigoid (herpes)gestationis (PG); (ii) polymorphic eruption of pregnancy (PEP); (iii) prurigo of pregnancy; and (iv) pruritic folliculitis of pregnancy (PF). The incidence of these specific disorders of pregnancy is 0.5 to $3.0 \%{ }^{14}$. In this study of 100 pregnant women, $10(10 \%)$ cases of specific dermatoses of pregnancy were seen. Of these the most common was PUPPP (polymorphic eruption of pregnancy) with a total of $(5 / 10)$ cases followed by 2 cases of pruritus gravidarum (intrahepatic cholestasis of pregnancy) and atopic eruption of pregnancy were seen in 3 cases. Of the disorders specific to pregnancy, 1 had prurigo of pregnancy, 1 had eczema in pregnancy and 1 had pruritic folliculitis of pregnancy. In a study done by Rashmi Kumar et al showed 22 cases of specific dermatoses out of 607 cases. Out of 22, 14 cases were found to have polymorphic eruption of pregnancy and 5 cases of pruritus gravidarum.

\section{CONCLUSION}

Awareness about and recognition of their clinical presentation is important for correct diagnosis that will direct the most appropriate laboratory evaluation and careful management in an effort to minimize maternal and fetal morbidity. From our study an unequivocal impression can be drawn, that pregnant women are prone to suffer from a wide range of dermatological problems and sexually transmitted diseases, apart from the specific dermatoses of pregnancy. In our study the physiological changes were more common as compared to the specific dermatoses of the pregnancy. Though physiological changes many times only need reassurance, as a physician we should not neglect performing thorough examination to distinguish physiological and other dermatoses of pregnancy as they might cause maternal and fetal morbidity and sometimes mortality and sometimes need early intervention.

\section{LIMITATIONS OF THE STUDY}

$\checkmark$ A much larger sample size would be clearly indicated to draw more valid inferences to the larger population.

$\$$ The incidence of the skin changes in pregnant women was not evaluated, only the patterns were described.

\section{REFERENCES}

1. Kroumpouzos G, Cohen LM. Dermatoses of pregnancy. Journal of the American Academy of Dermatology. 2001;45(1):1-19.

2. Winton $\mathrm{GB}$, Lewis $\mathrm{CW}$. Dermatoses of pregnancy. Journal of the American Academy of Dermatology. 1982;6(6):977-98.

3. Crawford GM, Leeper RW. Diseases of the skin in pregnancy. Archives of dermatology and syphilology. 1950;61(5):753-71.

4. Kumari R, Jaisankar TJ, Thappa DM. A clinical study of skin changes in pregnancy. Indian journal of dermatology, venereology and leprology. 2007;73(2):141.

5. Roger D, Vaillant L, Fignon A, Pierre F, Bacq Y, Brechot JF, et al. Specific pruritic diseases of pregnancy. A prospective study of 3192 pregnant women. Archives of dermatology. 1994;130(6):734-9.

6. Raj S KV, Kapasi A, et al. Skin in pregnancy. Indian J DermatolVenereolLeprol. 1992;58.

7. Shivakumar V MP. Skin in pregnancy. Indian journal of dermatology, venereology and leprology. 1999;65(1):235.

8. Wong RC, Ellis CN. Physiologic skin changes in pregnancy. Journal of the American Academy of Dermatology. 1984;10(6):929-40.

9. Poidevin LO. Striae gravidarum. Their relation to adrenal cortical hyperfunction. Lancet. 1959;2(7100):436-9.

10. Muzaffar F, Hussain I, Haroon TS. Physiologic skin changes during pregnancy: a study of 140 cases. International journal of dermatology. 1998;37(6):429-31

11. Martin AG, Leal-Khouri S. Physiologic skin changes associated with pregnancy. International journal of dermatology. 1992;31(6):375-8.

12. Esteve E, Saudeau L, Pierre F, Barruet K, Vaillant L, Lorette $G$. [Physiological cutaneous signs in normal pregnancy: a study of 60 pregnant women]. Annales de dermatologie et de venereologie. 1994;121(3):227-31.

13. Holmes RC, Black MM. The specific dermatoses of pregnancy. Journal of the American Academy of Dermatology. 1983;8(3):405-12.

14. Roger $D$, Vaillant L, Fignon A, Pierre F, Bacq $Y$, Brechot JF, et al. Specific pruritic diseases of pregnancy. A prospective study of 3192 pregnant women. Archives of dermatology. 1994;130(6):734-9. 